PROCEEDINGS OF THE AMERICAN MATHEMATICAL SOCIETY

Volume 124, Number 2, February 1996

\title{
SELF-SIMILAR SETS IN COMPLETE METRIC SPACES
}

\author{
ANDREAS SCHIEF
}

(Communicated by Christopher D. Sogge)

\begin{abstract}
We develop a theory for Hausdorff dimension and measure of selfsimilar sets in complete metric spaces. This theory differs significantly from the well-known one for Euclidean spaces. The open set condition no longer implies equality of Hausdorff and similarity dimension of self-similar sets and that $K$ has nonzero Hausdorff measure in this dimension. We investigate the relationship between such properties in the general case.
\end{abstract}

\section{INTRODUCTION}

In the study of fractal geometry, simple examples arise in the following manner: Given $N$ contracting similitudes $f_{i}, i=1, \ldots, N$, on a complete metric space $X$ there exists a unique nonempty compact $K$ such that $K=\bigcup_{i=1}^{N} f_{i}(K)$, a so-called self-similar fractal. The first observation in Euclidean spaces $X=\mathbb{R}^{s}$ is that the similarity dimension $\alpha$ and the Hausdorff dimension of $K$ (all terms are made precise in $\S 1)$ coincide if the "pieces" $f_{i}(K)$ are pairwise disjoint. This result remains true if the pieces have only "small overlap", sometimes called "just touching". To describe this separation property Hutchinson gave the following definition. The open set condition (OSC) is fulfilled if and only if there is a nonempty open set $G$ such that the sets $f_{i}(G)$ are disjoint and contained in $G$. Since the intersection of $G$ and $K$ may be empty, this condition is in general too weak to imply results concerning the dimension of the fractal. So Lalley strengthened the definition as follows: The strong open set condition (SOSC) holds if and only if furthermore $G \cap K \neq \varnothing$.

The following chain of implications holds in the Euclidean case and is best possible (cf. [6])

$$
\mathrm{SOSC} \Leftrightarrow \mathrm{OSC} \Leftrightarrow H^{\alpha}(K)>0 \Rightarrow \operatorname{dim} K=\alpha
$$

(by $H^{\alpha}$ we mean Hausdorff measure, by dim Hausdorff dimension).

This paper treats the general (non-Euclidean) case. We will see that the situation changes dramatically: The OSC ceases to be appropriate in this context, since it does not even imply $\operatorname{dim} K=\alpha$ (Example 3.1). The SOSC remains necessary for $H^{\alpha}(K)>0$ and sufficient only for $\operatorname{dim} K=\alpha$ (Theorem 2.6 and Example 3.2). The last example disproves a result in a paper of Stella ([7]), who claimed the equivalence of SOSC and $H^{\alpha}(K)>0$. His paper relied on the (false) statement

Received by the editors June 9, 1994 and, in revised form, August 23, 1994.

1991 Mathematics Subject Classification. Primary 28A80, 28A78.

Key words and phrases. SOSC, OSC, self-similar sets, fractals, Hausdorff dimension, complete metric spaces. 
that $K$ is a so-called $\beta$-space and on the correct observation (Theorem 2.4) that for $\beta$-spaces the SOSC implies $H^{\alpha}(K)>0$.

In [1] Bandt and Graf gave an algebraic characterization of $H^{\alpha}(K)>0$ for Euclidean spaces. Based on this paper we derived in [6] another necessary and sufficient condition ((iv) in (4.1)) for $H^{\alpha}(K)>0$ in these spaces. We show here in the general setting that this condition implies the SOSC (Theorem 2.1) and that $K$ is a $\beta$-space (Lemma 2.7), hence $H^{\alpha}(K)>0$ (Corollary 2.8). The reverse implication is derived in Theorem 2.9.

\section{NotATiOn AND DEFINITIONS}

We consider a topological space $X$ with complete metric $d$. The Hausdorff metric on closed subsets of $X$ is also denoted by $d$. We set $D(F, K)=\inf \{d(x, y): x \in$ $F, y \in K\}$ for subsets $F$ and $K$ of $X . A^{c}$ denotes the complement of $A, \bar{A}$ the closure. $U(\varepsilon, x)$ is the open $\varepsilon$-neighborhood of $x$ and $U(\varepsilon, F)=\bigcup\{U(\varepsilon, x): x \in F\}$. The Hausdorff measure of dimension $\alpha$ is denoted by $H^{\alpha}$.

Given $N \geq 2$ fix bijective similitudes $f_{1}, \ldots, f_{N}: X \rightarrow X$ with similarity ratios $\left.r_{i} \in\right] 0,1\left[\right.$, i.e. $d\left(f_{i}(x), f_{i}(y)\right)=r_{i} d(x, y)$ for $x, y \in X$. The self-similar set $K$ is the unique compact nonempty set such that

$$
K=\bigcup_{i=1}^{N} f_{i}(K)
$$

(see [4]).

We denote by $\mathcal{I}$ the set of all finite words $\mathbf{i}=\left(i_{1}, \ldots, i_{n}\right) \in\{1, \ldots, N\}^{n}$ with $n \geq 0$. The length of $\left(i_{1}, \ldots, i_{n}\right)$ is denoted by $|\mathbf{i}|=n$. Given $\mathbf{i}$ and $\mathbf{j}=\left(j_{1}, \ldots, j_{m}\right)$ we write $\mathbf{i} \mathbf{j}=\left(i_{1}, \ldots, i_{n}, j_{1}, \ldots, j_{m}\right)$. The words $\mathbf{i}$ and $\mathbf{j}$ are incomparable if there exists no $\mathbf{k}$ such that $\mathbf{i}=\mathbf{j} \mathbf{k}$ or $\mathbf{j}=\mathbf{i k}$.

We adopt the following conventions:

$$
\begin{aligned}
f_{\mathbf{i}} & =f_{i_{1}} \circ \cdots \circ f_{i_{n}}, \\
K_{\mathbf{i}} & =f_{\mathbf{i}}(K), \\
r_{\mathbf{i}} & =r_{i_{1}} \cdots r_{i_{n}} \\
r_{\mathbf{i}}^{*} & =r_{i_{1}} \cdots r_{i_{n-1}}
\end{aligned}
$$

Furthermore we set $r_{\max }=\max _{1 \leq i \leq N} r_{i}$ and $r_{\min }=\min _{1 \leq i \leq N} r_{i}$. We write $\alpha$ for the unique solution of $\sum_{i=1}^{N} r_{i}^{\alpha}=1$.

Finally we endow $\Omega=\{1, \ldots, N\}^{\mathbb{N}}$ with the product- $\sigma$-field and the canonical product measure

$$
\mathbb{P}=\otimes_{\mathbb{N}}\left(\sum_{i=1}^{N} r_{i}^{\alpha} \varepsilon_{i}\right),
$$

where $\varepsilon_{i}$ denotes the Dirac measure in point $i$. The image measure of $\mathbb{P}$ under the mapping from $\Omega$ to $K$ given by

$$
\pi:\left(i_{n}\right)_{n \in \mathbb{N}} \mapsto \lim _{n \rightarrow \infty} f_{\left(i_{1}, \ldots, i_{n}\right)}(x),
$$

which is independent of $x \in K$, will be called $\mu$. Write

$$
[\mathbf{i}]=\left\{\left(j_{n}\right) \in \Omega: i_{k}=j_{k}, k \leq|\mathbf{i}|\right\} .
$$


(1.1) Definition. We say that $\left(f_{1}, \ldots, f_{N}\right)$ (or, for brevity, $K$ ) fulfills the open set condition (OSC) if there exists a nonempty open set $G$ such that the sets $f_{i}(G)$, $1 \leq i \leq N$, are pairwise disjoint and all contained in $G$. If $G \cap K \neq \varnothing$ the strong OSC (SOSC) is fulfilled.

Next, since the constants $r_{i}$ may be different, we have to give the following definition: Given $1 \geq b>0$ we set

$$
\mathcal{I}_{b}=\left\{\mathbf{i}=\left(i_{1}, \ldots, i_{n}\right): r_{\mathbf{i}}<b \leq r_{\mathbf{i}}^{*}\right\}
$$

(for the sake of completeness $\mathcal{I}_{b}=\{\varnothing\}$ if $b>1$ ). The elements of $\mathcal{I}_{b}$ are obviously incomparable and satisfy

$$
K=\bigcup_{\mathbf{i} \in \mathcal{I}_{b}} K_{\mathbf{i}}
$$

where the $K_{\mathbf{i}}$ are copies of $K$ of "approximately equal size".

Let $\varepsilon>0$. We write

$$
\begin{gathered}
G_{\mathbf{k}}=U\left(\varepsilon r_{\mathbf{k}}, K_{\mathbf{k}}\right), \\
\mathcal{I}(\mathbf{k})=\left\{\mathbf{i} \in \mathcal{I}_{\operatorname{diam} G_{\mathbf{k}}}: K_{\mathbf{i}} \cap G_{\mathbf{k}} \neq \varnothing\right\}
\end{gathered}
$$

and

$$
\gamma_{\varepsilon}=\sup _{\mathbf{k}} \# \mathcal{I}(\mathbf{k})
$$

(where $\# C$ denotes the cardinality of $C$ ). These terms have been used by Bandt and Graf in [1], where it is shown that $\gamma_{\varepsilon}<\infty$ is implied by $H^{\alpha}(K)>0$ if $X$ is Euclidean.

\section{THEOREMS}

Note first that $H^{\alpha}(K)<\infty$ without any further assumption (cf. [4]). We start with an observation from [6].

(2.1) Theorem. If $\gamma_{\varepsilon}<\infty$ for some $\varepsilon>0$ the SOSC is fulfilled.

Proof. 1. Choose $\mathbf{k}$ such that $\gamma_{\varepsilon}=\# \mathcal{I}(\mathbf{k})$. We show for arbitrary $\mathbf{j}$

$$
\mathcal{I}(\mathbf{j k})=\{\mathbf{j i}: \mathbf{i} \in \mathcal{I}(\mathbf{k})\} .
$$

By maximality, we only have to show " $\supset$ ". But this is clear since $\varnothing \neq K_{\mathbf{i}} \cap G_{\mathbf{k}}$ implies

$$
\begin{aligned}
\varnothing & \neq f_{\mathbf{j}}\left(K_{\mathbf{i}} \cap G_{\mathbf{k}}\right) \\
& =f_{\mathbf{j}}\left(K_{\mathbf{i}}\right) \cap f_{\mathbf{j}}\left(G_{\mathbf{k}}\right) \\
& =K_{\mathbf{j} \mathbf{i}} \cap f_{\mathbf{j}}\left(U\left(\varepsilon r_{\mathbf{k}}, K_{\mathbf{k}}\right)\right) \\
& =K_{\mathbf{j} \mathbf{i}} \cap U\left(\varepsilon r_{\mathbf{j k}}, K_{\mathbf{j k}}\right) \\
& =K_{\mathbf{j} \mathbf{i}} \cap G_{\mathbf{j k}} .
\end{aligned}
$$

Now let $l \neq j_{1}$ (if $\mathbf{j}=\varnothing$ nothing remains to be shown). Since $K_{l}$ is covered by $\left\{K_{\mathbf{l}}: \mathbf{l} \in \mathcal{I}_{\text {diam } G_{\mathrm{jk}}}, l_{1}=l\right\}$, we get immediately

$$
D\left(K_{\mathbf{j k}}, K_{l}\right) \geq \varepsilon r_{\mathbf{j k}} .
$$

2. Now we are in a position to define the desired separating open set:

$$
U=\bigcup_{\mathbf{j} \in \mathcal{I}} G_{\mathbf{j k}}^{*}
$$


where $G_{\mathbf{i}}^{*}=U\left(\varepsilon r_{\mathbf{i}} / 2, K_{\mathbf{i}}\right)$. We get

$$
K_{\mathbf{k}} \subset G_{\mathbf{k}}^{*} \subset U
$$

so for each $i$ (cf. part 1)

$$
f_{i}(U)=\bigcup_{\mathbf{j} \in \mathcal{I}} f_{i}\left(G_{\mathbf{j k}}^{*}\right)=\bigcup_{\mathbf{j} \in \mathcal{I}} G_{i \mathbf{j k}}^{*} \subset U
$$

and finally for each $i \neq j$

$$
f_{i}(U) \cap f_{j}(U)=\varnothing .
$$

For otherwise, there are $\mathbf{i}$ and $\mathbf{j}$ such that $G_{i \mathbf{i k}}^{*} \cap G_{j \mathbf{j k}}^{*} \neq \varnothing$ and $r_{i \mathbf{i k}} \geq r_{j \mathbf{j k}}$. If $y$ is an element of this intersection there exist $y_{1} \in K_{i \mathbf{i k}}$ and $y_{2} \in K_{j \mathbf{j k}}$ such that $d\left(y, y_{1}\right)<\varepsilon r_{i \mathbf{i k}} / 2$ and $d\left(y, y_{2}\right)<\varepsilon r_{j \mathbf{j k}} / 2$. We get $d\left(y_{1}, y_{2}\right)<\varepsilon r_{i \mathbf{i k}}$, hence $D\left(K_{i \mathbf{i k}}, K_{j}\right)<\varepsilon r_{i \mathbf{i k}}$ which contradicts part 1 .

The following definition was given by Larman in [5].

(2.2) Definition. If $B \subset X$ and $\rho>0$ we denote by $N(B, \rho)$ the (possibly infinite) maximal number of disjoint closed balls with radius $\rho$ and centers in $B$. If for each $0<\beta<1$ there are constants $C$ and $D$ such that $N(U(R, x), \beta R)<C$ holds for each $D>R>0$ and $x \in B$ we call $B$ an $\beta$-space.

(2.3) Corollary. Each Euclidean space $\mathbb{R}^{s}$ is a $\beta$-space. Each compact subset of a $\beta$-space is a $\beta$-space.

Proof. For the first assertion use Lebesgue measure to calculate $N(B, \rho)$. The second one is an immediate consequence of Theorem 11 in [5].

The following theorem is well known for Euclidean spaces and was claimed in [7] by Stella. We give a different proof.

(2.4) Theorem. If $K$ is a $\beta$-space which fulfills the SOSC, then $H^{\alpha}(K)>0$.

Proof. Let $G$ be an open set given by the SOSC for $K, x \in K$ and $\varepsilon>0$ such that $U(\varepsilon, x) \subset G$. Let $B \subset X$ be measurable such that $b=\operatorname{diam} B / \operatorname{diam} K \leq 1$. Set $\mathcal{I}=\left\{\mathbf{i} \in \mathcal{I}_{b}: K_{\mathbf{i}} \cap B \neq \varnothing\right\}$. The balls $f_{\mathbf{i}}(U(\varepsilon, x)) \cap K=U\left(\varepsilon r_{\mathbf{i}}, f_{\mathbf{i}}(x)\right) \cap K, \mathbf{i} \in \mathcal{I}$, are pairwise disjoint and for any $y \in B$ contained in

$$
U\left(\operatorname{diam} B+\max _{\mathbf{i} \in \mathcal{I}} \operatorname{diam} K_{\mathbf{i}}+\max _{\mathbf{i} \in \mathcal{I}} \varepsilon r_{\mathbf{i}}, y\right) \subset U(b(2 \operatorname{diam} K+\varepsilon), y) .
$$

So (setting $\beta=\frac{\varepsilon r_{\min }}{2 \operatorname{diam} K+\varepsilon}$ ) there is a constant $C$ only depending on $\varepsilon$ such that $\# \mathcal{I} \leq C$ and therefore

$$
\mu(B) \leq C \max _{i \in \mathcal{I}} \mu\left(K_{\mathbf{i}}\right) \leq C b^{\alpha}=C(\operatorname{diam} K)^{\alpha}(\operatorname{diam} B)^{\alpha} .
$$

By the mass distribution principle $([3], 4.2)$ the proof is complete.

In the general setting we first consider the following simple case.

(2.5) Theorem. If $K_{1}, \ldots, K_{N}$ are pairwise disjoint, then $H^{\alpha}(K)>0$.

Proof. Let $\delta=\min _{i \neq j} D\left(K_{i}, K_{j}\right)$. By induction we have immediately that

$$
D\left(K_{\mathbf{i}}, K_{\mathbf{j}}\right) \geq \delta r_{\mathbf{i}}^{*}
$$


if $\mathbf{i}$ and $\mathbf{j}$ are incomparable. Again consider a measurable set $B \subset X$ such that $b=2 \operatorname{diam} B / \delta \leq 1$. Then, since $\operatorname{diam} B<\delta r_{\mathbf{i}}^{*}$, at most one set $K_{\mathbf{i}}, \mathbf{i} \in \mathcal{I}_{b}$, meets $B$, and therefore

$$
\mu(B) \leq \mu\left(K_{\mathbf{i}}\right)=r_{\mathbf{i}}^{\alpha}<b^{\alpha}=2^{\alpha} \delta^{-\alpha}(\operatorname{diam} B)^{\alpha} .
$$

Again the result follows by the mass distribution principle.

This theorem has the following consequences.

(2.6) Theorem. The SOSC implies that $\operatorname{dim} K=\alpha$.

Proof. Let $G$ be an open set given by the SOSC for $K$. There exist $x \in G \cap K$ and an index $\mathbf{i}$ such that $x \in K_{\mathbf{i}} \subset G$. So, given an arbitrary integer $n$, the sets $K_{\mathbf{j i}}$, $|\mathbf{j}|=n$, are pairwise disjoint. The self-similar set corresponding to the mappings $f_{\mathbf{j i}},|\mathbf{j}|=n$, fulfills the assumptions of Theorem 2.5 and we get

$$
\alpha_{n} \leq \operatorname{dim} K \leq \alpha
$$

where $\alpha_{n}$ satisfies

$$
1=\sum_{|\mathbf{j}|=n} r_{\mathbf{j} \mathbf{i}}^{\alpha_{n}}=r_{\mathbf{i}}^{\alpha_{n}} \sum_{|\mathbf{j}|=n} r_{\mathbf{j}}^{\alpha_{n}}
$$

In order to derive a contradiction let us assume $\beta:=\operatorname{dim} K<\alpha$. We get

$$
r_{\mathbf{i}}^{-\alpha_{n}}=\sum_{|\mathbf{j}|=n} r_{\mathbf{j}}^{\alpha_{n}} \geq \sum_{|\mathbf{j}|=n} r_{\mathbf{j}}^{\beta}=\sum_{|\mathbf{j}|=n} r_{\mathbf{j}}^{\alpha} r_{\mathbf{j}}^{\beta-\alpha} \geq \sum_{|\mathbf{j}|=n} r_{\mathbf{j}}^{\alpha} r_{\max }^{n(\beta-\alpha)}=r_{\max }^{n(\beta-\alpha)}
$$

But the last term tends to infinity as $n$ tends to infinity, which is impossible since $\alpha_{n}$ is bounded by $\beta$.

The following lemma will be used in the following corollary.

(2.7) Lemma. If $\gamma_{\varepsilon}<\infty$ for some $\varepsilon>0$, then $K$ is a $\beta$-space.

Proof. 1. We first prove for $\rho \leq \operatorname{diam} K$ that

$$
N(K, \rho) \leq\left(\frac{\operatorname{diam} K}{\rho r_{\min }}\right)^{\alpha} .
$$

Let $B_{1}, \ldots, B_{p}$ be disjoint balls with radius $\rho$ and centers $x_{k}$ in $K$. There are $\mathbf{i}_{k} \in$ $\mathcal{I}_{\rho / \operatorname{diam} K}$ such that $x_{k} \in K_{\mathbf{i}_{k}}$. Since obviously $K_{\mathbf{i}_{k}} \subset B_{k}$, these sets are pairwise disjoint and the self-similar set corresponding to the mappings $f_{\mathbf{i}_{k}}, k=1, \ldots, p$, fulfills the assumptions of (2.5). If the dimension of this set is $\beta$ we get $\beta \leq \alpha$ and $\sum_{k=1}^{p} r_{\mathbf{i}_{k}}^{\beta}=1$ and therefore

$$
1=\sum_{k=1}^{p} r_{\mathbf{i}_{k}}^{\beta} \geq p\left(\frac{\rho r_{\min }}{\operatorname{diam} K}\right)^{\beta} \geq p\left(\frac{\rho r_{\min }}{\operatorname{diam} K}\right)^{\alpha},
$$

the desired inequality.

2. Let $x \in K$ and $R, \rho$ be given. Choose $\mathbf{i} \in \mathcal{I}_{b}, b=R / \varepsilon$, such that $x \in K_{\mathbf{i}}$. It follows that

$$
U(R, x) \subset U\left(\varepsilon r_{\mathbf{i}}^{*}, K_{\mathbf{i}}\right)
$$


It suffices to count the number of disjoint balls with centers in the second set. To this end we consider the corresponding number for the sets $K_{\mathbf{k}}, \mathbf{k} \in \mathcal{I}(\mathbf{i})$, which is easy to calculate by part 1 and the self-similarity of $K$. Thus

$$
N(U(R, x), \rho) \leq \sum_{\mathbf{k} \in \mathcal{I}(\mathbf{i})}\left(\frac{\operatorname{diam} K_{\mathbf{k}}}{\rho r_{\min }}\right)^{\alpha} \leq \gamma_{\varepsilon} C(R / \rho)^{\alpha}
$$

for $C$ not depending on $R$ and $\rho$.

(2.8) Corollary. If $\gamma_{\varepsilon}<\infty$ for some $\varepsilon>0$, then $H^{\alpha}(K)>0$.

Proof. Just combine (2.7) with (2.1) and (2.4).

We now prove the reverse implication. For Euclidean spaces this has been shown in [1].

(2.9) Theorem. If $H^{\alpha}(K)>0$, then $\gamma_{\varepsilon}<\infty$ for arbitrary $\varepsilon>0$.

Proof. 1. If we denote by $\nu=\left.\left(H^{\alpha}(K)\right)^{-1} H^{\alpha}\right|_{K}$ the normalized restriction of Hausdorff measure to the set $K$ we observe first that $\nu=\mu$. Indeed, self-similarity yields at once

$$
\nu\left(K_{\mathbf{i}}\right)=\frac{H^{\alpha}\left(K_{\mathbf{i}}\right)}{H^{\alpha}(K)}=r_{\mathbf{i}}^{\alpha}=\mu\left(K_{\mathbf{i}}\right)
$$

for arbitrary i. The last equality is due to S. Graf: Assume that there is an $\mathbf{i}_{0}$ with $\mu\left(K_{\mathbf{i}_{0}}\right)>r_{\mathbf{i}_{0}}^{\alpha}$. Then there is a word $\mathbf{j}_{0}$ different from $\mathbf{i}_{0}$ but of same length with

$$
\mathbb{P}\left(\left[\mathbf{j}_{0}\right] \cap \pi^{-1}\left(K_{\mathbf{i}_{0}}\right)\right)>0 .
$$

Set

$$
U=\Omega \backslash\left(\left[\mathbf{j}_{0}\right] \cap \pi^{-1}\left(K_{\mathbf{i}_{0}}\right)\right) .
$$

Then $U$ is open and $\pi(U)=K$. There is a sequence $\mathbf{i}_{n}$ of words with

$$
U=\bigcup_{n \in \mathbb{N}}\left[\mathbf{i}_{n}\right]
$$

and $\left[\mathbf{i}_{n}\right] \cap\left[\mathbf{i}_{m}\right]=\varnothing$ for $n \neq m$. Then for each $k \in \mathbb{N}$,

$$
K \subset \bigcup_{n_{1} \in \mathbb{N}} \cdots \bigcup_{n_{k} \in \mathbb{N}} K_{\mathbf{i}_{n_{1}}} \cdots \mathbf{i}_{n_{k}}
$$

hence

$$
H^{\alpha}(K) \leq H^{\alpha}(K) \sum_{n_{1} \in \mathbb{N}} \cdots \sum_{n_{k} \in \mathbb{N}} r_{\mathbf{i}_{n_{1}}}^{\alpha} \cdots r_{\mathbf{i}_{n_{k}}}^{\alpha} \rightarrow 0
$$

a contradiction.

2. Let $G$ be nonempty and open in $K$. We show that

$$
H^{\alpha}(G) \leq(\operatorname{diam} G)^{\alpha} .
$$

Let $F$ be the closure of $G$. Let $C=\frac{H^{\alpha}(F)}{(\operatorname{diam} F)^{\alpha}}$ (if diam $F=0$ there is nothing further to be shown). It suffices to show $C \leq 1$. Let $\mathbf{i}$ be a word such that $K_{\mathbf{i}} \subset G \subset F$. Obviously

$$
\mathbb{P}\left(\left\{\left(i_{n}\right): \mathbf{i}=\left(i_{k+1}, i_{k+2}, \ldots, i_{k+|\mathbf{i}|}\right) \text { for infinitely many } k\right\}\right)=1,
$$


which implies $\mu(L)=1$ for

$$
L=\bigcap \bigcup_{k} \bigcup_{l \geq k|\mathbf{j}|=l} K_{\mathbf{j i}}
$$

So $\mathcal{V}=\left\{f_{\mathbf{j}}(F): \mathbf{j}\right.$ a word $\}$ is a Vitali class for $L$. Theorem 1.10 in [2] (generalized to arbitrary metric spaces) therefore yields $\mathbf{j}_{1}, \mathbf{j}_{2}, \ldots$ such that the sets $f_{\mathbf{j}_{k}}(F)$ are pairwise disjoint and one of the following cases holds.

First case: $\sum_{k}\left(\operatorname{diam}\left(f_{\mathbf{j}_{k}}(F)\right)^{\alpha}=\infty\right.$.

Since self-similarity gives $H^{\alpha}\left(f_{\mathbf{j}_{k}}(F)\right)=C\left(\operatorname{diam} f_{\mathbf{j}_{k}}(F)\right)^{\alpha}$, this would imply $H^{\alpha}(K) \geq H^{\alpha}(L)=\infty$ giving a contradiction.

Second case: $H^{\alpha}\left(L \backslash L^{\prime}\right)=0$ for $L^{\prime}=\bigcup_{k} f_{\mathbf{j}_{k}}(F)$.

Since by part $1 H^{\alpha}(L)=H^{\alpha}(K)$, it follows that $H^{\alpha}\left(L^{\prime}\right)>0$. Proposition 3 of [1] yields

$$
H^{\alpha}\left(L^{\prime}\right) \leq \sum_{k}\left(\operatorname{diam} f_{\mathbf{j}_{k}}(F)\right)^{\alpha} .
$$

From the above this sum is equal to

$$
\sum_{k} C^{-1} H^{\alpha}\left(f_{\mathbf{j}_{k}}(F)\right)=C^{-1} H^{\alpha}\left(L^{\prime}\right),
$$

so $C \leq 1$.

3. Let $\mathbf{k}$ be given. We check the cardinality of $\mathcal{I}(\mathbf{k})$. By Proposition 2 of [1] the sets $K_{\mathbf{i}}, \mathbf{i} \in \mathcal{I}(\mathbf{k})$, are pairwise $\mu$-almost disjoint, and therefore by part 1 also $H^{\alpha}$-almost disjoint. This together with part 2 yields

$$
\begin{aligned}
\sum_{\mathbf{i} \in \mathcal{I}(\mathbf{k})} H^{\alpha}\left(K_{\mathbf{i}}\right) & \leq H^{\alpha}\left(U\left(\max _{\mathbf{i} \in \mathcal{I}(\mathbf{k})} \operatorname{diam} K_{\mathbf{i}}, G_{\mathbf{k}}\right) \cap K\right) \\
& \leq(b+2 b \operatorname{diam} K)^{\alpha},
\end{aligned}
$$

where $b=\operatorname{diam} G_{\mathbf{k}}$. The left-hand side of this inequality is surely greater or equal to

$$
\# I(\mathbf{k}) H^{\alpha}(K) b^{\alpha} r_{\min }^{\alpha},
$$

which implies

$$
\gamma_{\varepsilon} \leq \frac{(1+2 \operatorname{diam} K)^{\alpha}}{H^{\alpha}(K) r_{\min }^{\alpha}}
$$

\section{EXAMPLES}

The first example shows that in the general case the open set condition does not imply any useful conclusion.

(3.1) Example. There exist self-similar sets $K$ of dimension 0 which fulfill the OSC.

Proof. Let $X=\{(x, n): x \neq 0$ real, $n \in \mathbb{Z}\} \cup\{0\}$ ( $\mathbb{Z}$ denotes the integers) be endowed with the complete metric $d$ defined by

$$
\begin{aligned}
d(0,(x, n)) & =|x|, \\
d((x, n),(y, n)) & =|x-y|, \\
d((x, n),(y, m)) & =|x|+|y|
\end{aligned}
$$


if $n \neq m$. We define the similitudes $f_{1}$ and $f_{2}$ by

$$
f_{i}(0)=0
$$

and

$$
f_{i}(x, n)=\left(x / 2, F_{i}(n)\right)
$$

where

$$
\begin{gathered}
F_{1}(n)= \begin{cases}2 n & \text { if } n \geq 0, \\
-n & \text { if } n<0 \text { odd } \\
n / 2 & \text { if } n<0 \text { even, }\end{cases} \\
F_{2}(n)= \begin{cases}2 n-1 & \text { if } n>0, \\
-n & \text { if } n \leq 0 \text { even } \\
(n-1) / 2 & \text { if } n<0 \text { odd. }\end{cases}
\end{gathered}
$$

We get at once $r_{1}=r_{2}=1 / 2$ and $\alpha=1$, but $K=\{0\}$. Furthermore the open set $G=\{(x, n): x>0, n>0\}$ satisfies the OSC.

The following example shows that there exist self-similar sets which are not $\beta$ spaces, and even worse, that the SOSC does not suffice to give $H^{\alpha}(K)>0$ in the general case.

(3.2) Example. There exists a self-similar set $K \subset l_{1}$ fulfilling the SOSC, but with $H^{\alpha}(K)=0$.

Proof. We consider $l_{1}=\left\{\left(x_{z}\right) \in \mathbb{R}^{\mathbb{Z}}: \sum_{z \in \mathbb{Z}}\left|x_{z}\right|<\infty\right\}$ endowed with the $l_{1}$-norm. The symbols $e_{n}$ denote the usual basis of $l_{1}$. We define the mappings $f_{1}$ and $f_{2}$ by

$$
f_{i}\left(\sum x_{z} e_{z}\right)=\sum \frac{x_{z}}{2} e_{\pi_{i}(z)}
$$

where $\pi_{i}$ are arbitrary bijections of the integers such that $\pi_{i}(n)=2 n+i$ for $n \geq 0$. The third mapping is defined simply by

$$
f_{3}(x)=e_{0}+\frac{1}{2}\left(x-e_{0}\right) .
$$

We have $r_{1}=r_{2}=r_{3}=1 / 2$.

1. To prove the SOSC just look at the following disjoint union of open sets:

$$
G=\bigcup_{k=0}^{\infty} U\left(2^{-n_{k}}, 2^{-n_{k}} e_{k}\right)
$$

where $n_{0}=1, n_{1}=n_{2}=2, n_{3}=\cdots=n_{6}=3, \ldots, n_{2^{k-1}-1}=\cdots=n_{2^{k}-2}=k, \ldots$. Since the point $f_{3}\left(f_{1}\left(e_{0}\right)\right)=\frac{1}{2} e_{0}+\frac{1}{4} e_{1}$ is contained in $G$, the intersection $G \cap K$ is nonempty. We calculate the images of $G$ :

$$
f_{i}(G)=\bigcup_{k=0}^{\infty} U\left(2^{-n_{k}-1}, 2^{-n_{k}-1} e_{2 k+i}\right)=\bigcup_{k=0}^{\infty} U\left(2^{-n_{2 k+i}}, 2^{-n_{2 k+i}} e_{2 k+i}\right),
$$

for $i=1,2$, and

$$
f_{3}(G) \subset f_{3}(U(1,0))=U\left(1 / 2,1 / 2 e_{0}\right) .
$$

These sets are disjoint and contained in $G$.

2. Let $k$ be a positive integer and $\mathcal{I}_{k}=\{1,2\}^{k}$. Let

$$
\mathcal{I}_{k}^{0}=\left\{\left(i_{1}, \ldots, i_{p}\right): p \geq 1, i_{p}=3,\left(i_{l}, \ldots, i_{l+k-1}\right) \notin \mathcal{I}_{k} \text { for each } l\right\} \cup\{\varnothing\} .
$$


The following union is disjoint:

$$
\Omega_{k}=\bigcup_{\mathbf{j} \in \mathcal{I}_{k}} \bigcup_{\mathbf{i} \in \mathcal{I}_{k}^{0}}[\mathbf{i j}] .
$$

Since, if not, there are $\mathbf{i}, \mathbf{i}^{\prime} \in \mathcal{I}_{k}^{0}, \mathbf{j}, \mathbf{j}^{\prime} \in \mathcal{I}_{k}$ and $\mathbf{k}$ such that

$$
\mathbf{i j k}=\mathbf{i}^{\prime} \mathbf{j}^{\prime} \text {. }
$$

But $\mathbf{j}$ consists only of elements 1 or 2 and the last element of $\mathbf{i}^{\prime}$ is 3 . So either $\mathbf{j}$ is contained in $\mathbf{i}^{\prime}$ (in contradiction to the construction of $\mathcal{I}_{k}^{0}$ ) or it is equal to $\mathbf{j}^{\prime}$ and $\mathbf{k}=\varnothing$.

In addition, $\mathbb{P}\left(\Omega_{k}\right)=1$ since for $\mathbb{P}$-almost all elements $\left(i_{1}, i_{2}, \ldots\right)$ of $\Omega$ there is a first sequence $\left(3, j_{1}, \ldots, j_{k}\right)$, where the $j_{m}$ are 1 or 2 .

By calculating the corresponding $\mathbb{P}$-measures we get

$$
1=\mathbb{P}\left(\Omega_{k}\right)=\sum_{\mathbf{j} \in \mathcal{I}_{k}} \sum_{\mathbf{i} \in \mathcal{I}_{k}^{0}} r_{\mathbf{i}}^{\alpha} r_{\mathbf{j}}^{\alpha}=2^{k}\left(2^{-k}\right)^{\alpha} \sum_{\mathbf{i} \in \mathcal{I}_{k}^{0}} r_{\mathbf{i}}^{\alpha} .
$$

Another consequence is $\mu\left(L_{k}\right)=1$ where

$$
L_{k}=\bigcup_{\mathbf{j} \in \mathcal{I}_{k}} \bigcup_{\mathbf{i} \in \mathcal{I}_{k}^{0}} K_{\mathbf{i j}}
$$

Therefore $H^{\alpha}\left(L_{k}\right)=H^{\alpha}(K)$ since either $H^{\alpha}(K)=0$ or otherwise, by part 1 of the proof of $(2.9), \mu\left(K \backslash L_{k}\right)=0$ implies $H^{\alpha}\left(K \backslash L_{k}\right)=0$.

3. Since $K \subset \bar{G}$ (cf. [4]), we see by part 1 that

$$
\bigcup_{\mathbf{j} \in \mathcal{I}_{k}} K_{\mathbf{j}} \subset \bigcup_{\mathbf{j} \in \mathcal{I}_{k}} \overline{f_{\mathbf{j}}(G)} \subset \overline{U\left(2^{-k}, 0\right)} .
$$

Hence

$$
L_{k} \subset \bigcup_{\mathbf{i} \in \mathcal{I}_{k}^{0}} \overline{f_{\mathbf{i}}\left(U\left(2^{-k}, 0\right)\right)}
$$

So we get by Proposition 3 in [1] and part 2

$$
H^{\alpha}(K)=H^{\alpha}\left(L_{k}\right) \leq \sum_{\mathbf{i} \in \mathcal{I}_{k}^{0}}\left(2^{1-k} r_{\mathbf{i}}\right)^{\alpha}=2^{\alpha(1-k)} 2^{-k} 2^{\alpha k}=2^{\alpha-k},
$$

which tends to zero as $k$ tends to infinity.

The last example is due to Mattila (personal communication):

(3.3) Example. There are self-similar sets $K$ of dimension $\alpha$ which do not fulfill the SOSC.

Proof. Take a nonconnected Sierpinski gasket in $\mathbb{R}^{2}$, i.e. for $i=1,2,3$ set $f_{i}(x)=$ $x_{i}+\frac{x-x_{i}}{3}$ with $x_{1}=0, x_{2}=(1,0)$ and $x_{3}=(1 / 2,1 / 2 \sqrt{3})$. This fractal obviously fulfills the SOSC with $\alpha=1$. Therefore (see [2]) Lebesgue almost all projections onto one-dimensional subspaces of $\mathbb{R}^{2}$ (which are again self-similar) have dimension 1 but zero Lebesgue measure. So the SOSC cannot hold by Theorem 2.4. 


\section{Conclusion}

Consider the following statements:

(i) The sets $K_{1}, \ldots, K_{N}$ are disjoint,

(ii) $H^{\alpha}(K)>0$,

(iii) for each $\varepsilon>0, \gamma_{\varepsilon}<\infty$ holds,

(iv) there exists $\varepsilon>0$ such that $\gamma_{\varepsilon}<\infty$,

(v) SOSC,

(vi) the dimension of $K$ is $\alpha$.

We conclude this paper by the following theorem.

(4.1) Theorem. The following list of implications and equivalences is valid for complete metric spaces. No other implications are valid in general:

$$
\text { (i) } \Rightarrow \text { (ii) } \Leftrightarrow \text { (iii) } \Leftrightarrow \text { (iv) } \Rightarrow(\mathrm{v}) \Rightarrow \text { (vi). }
$$

If $K$ is a $\beta$-space, which is always the case if $X$ is a $\beta$-space, the statements (iv) and $(\mathrm{v})$ are also equivalent.

Proof. See above.

\section{REFERENCES}

1. C. Bandt and S. Graf, Self-similar sets 7. A characterization of self-similar fractals with positive Hausdorff measure, Proc. Amer. Math. Soc. 114 (1992), 995-1001. MR 93d:28014

2. K. J. Falconer, The geometry of fractal sets, Cambridge Univ. Press, Cambridge and New York, 1986. MR 88d:28001

3. _ Fractal geometry, Wiley, New York, 1990. MR 92j:82053

4. J. E. Hutchinson, Fractals and self-similarity, Indiana Univ. Math. J. 30 (1981), 713-747.

5. D. J. Larman, A new theory of dimension, Proc. London Math. Soc. (3) 17 (1967), 178-192. MR 34:3540

6. A. Schief, Separation properties for self-similar sets, Proc. Amer. Math. Soc. 122 (1994), 111115. MR 94k:28012

7. S. Stella, On Hausdorff dimension of recurrent net fractals, Proc. Amer. Math. Soc. 116 (1992), 389-400; corrigendum, 121 (1994), 1309-1311. MR 92m:58079; MR 95g:58141

Corporate Research and Development, Siemens AG, 81730, Munich, Germany

E-mail address: andreas.schief@zfe.siemens.de 